\title{
Vers une anthropologie de la corruption : les histoires tragiques de Jean-Pierre Camus
}

Joël Zufferey

\section{(2) OpenEdition \\ Journals}

Édition électronique

URL : http://journals.openedition.org/edl/912

DOI : $10.4000 /$ edl. 912

ISSN : 2296-5084

Éditeur

Université de Lausanne

\section{Édition imprimée}

Date de publication : 15 décembre 2015

ISBN : 978-2-940331-47-5

ISSN : 0014-2026

\section{Référence électronique}




\section{VERS UNE ANTHROPOLOGIE DE LA CORRUPTION : LES HISTOIRES TRAGIQUES DE JEAN-PIERRE CAMUS}

La présente étude vise à montrer que, par la syntagmatique particulière qu'il confère à chaque récit, Jean-Pierre Camus réserve un traitement systématique aux nombreux cas de corruption qu'il met en scène dans ses nouvelles tragiques. L'analyse, menée dans une perspective linguistique, abordera plusieurs niveaux de la narrativité: la désignation des agents et son évolution au cours du récit, mais aussi l'expression de l'intentionnalité qui préside à l'action constituent les lieux discursifs par lesquels se distinguent des modes de corruption. L'agencement varié de ces composantes narratives donne lieu, dès lors qu'elles sont envisagées à l'échelle du recueil, à ce que nous appelons une anthropologie de la corruption.

C'est de la cacochimie de nostre humeur depravée que nous contournons comme serpens en venin les viandes plus salutaires, \& comme si nous avions l'attouchement contagieux, nous corrompons par nostre maniement ce qui est bon de soy.

J.-P. Camus, «De la Corruption», 1610.

"Corruption" est avant tout un signe linguistique, c'est-à-dire l'expression d'un sens. Et l'on sait, avec Ferdinand de Saussure, que la forme et l'idée du mot concourent à leur institution réciproque: la matérialité sensorielle (ou image acoustique) configure le concept qui, dans un même temps, délimite la forme. La corruption trouve ainsi en relation de voisinage avec d'autres lexèmes tels que l'altération, la décomposition, la dissolution, etc. - une place originale dans le vocabulaire français. Ces principes de sémiologie pourraient ouvrir la voie à une investigation lexicologique, centrée sur le système de la langue à une époque donnée. Notre étude, attachée à décrire les formes linguistiques, 
sera cependant d'un autre ordre dans la mesure où nous proposons d'examiner la pertinence sémantique du terme en discours, dans les nouvelles de l'évêque Jean-Pierre Camus (1584-1652) en particulier. Nous adopterons, comme support de l'analyse, le premier des vingt et un recueils publiés par l'auteur, Les Evenemens singuliers (1628). Procéder à l'analyse d'un corpus s'impose à notre démarche dès lors que nous ne chercherons pas à nuancer ou enrichir spéculativement le concept. Certes, il peut être légitime d'appréhender la corruption en tant que processus général et de réfléchir sur les causes, les conséquences ou les phases de son développement telles qu'elles se trouvent débattues par plusieurs auteurs d'une même époque. Mais plutôt que de dégager un sens général, même historicisé, de la corruption, nous voulons montrer qu'un discours narrant les méfaits d'un personnage contribue, par sa syntagmatique particulière, à construire et définir la notion.

Dans cette optique la corruption consiste, au départ de l'analyse, en une formule quasiment vide, étant établi qu'une signification a priori ne nous intéresse guère. C'est alors le discours - non pas tant celui qui, métalinguistiquement ou philosophiquement, parle à son propos, mais celui qui dit ou montre la corruption en acte - qui se charge de sémantiser l'expression ${ }^{1}$. Nous dégagerons donc certaines composantes sémantiques de la corruption en faisant porter notre attention sur les formes discursives que réalise Camus pour figurer des individus malfaisants, et ce n'est qu'en soutien à l'analyse qu'il nous arrivera de recourir à d'éventuels commentaires de l'auteur thématisant l'objet. Par cette démarche, nous nous proposons d'exploiter la transitivité syntaxique du nom: en effet, la corruption n'existe pas sans objet, elle est toujours corruption de quelque chose ou de quelqu'un. L'opération de détermination ou de spécification du processus, appelée par la préposition (corruption de...), associe la corruption à un objet, mais ancre aussi le phénomène dans un état de fait et ainsi le particularise. Partant, les moyens discursifs par lesquels Camus attribue un substrat au mécanisme de la corruption vont nous permettre d'en saisir certains fonctionnements et enjeux de sens pertinents dans le cadre idéologique de l'auteur. La confrontation de nos résultats à d'autres nouvelles, voire à d'autres pratiques discursives,

I. Une présomption de signification, que les dictionnaires établissent abstraitement, apparaît naturellement indispensable pour reconnaître les phénomènes de corruption dans des textes qui représentent la corruption sans forcément la nommer. 
produites à l'époque permettrait de mesurer avec finesse, à travers les façons de dire la corruption plus que par ce qui en est dit, la conformité des représentations camusiennes à celles de son temps. Cette dernière tâche excède les ambitions de ce travail et ne sera pas conduite ici.

\section{La désignation de l'agent}

Dans l'une de ses réalisations grammaticales, la corruption apparaît comme médiation entre deux instances: $\mathrm{X}$ corrompt $\mathrm{Y}$. Ce mode transactionnel n'est pas ignoré des $\mathrm{XVI}^{\mathrm{e}}$ et $\mathrm{XVII}{ }^{\mathrm{e}}$ siècles, ainsi que le met en évidence l'étude de Jean-Claude Waquet qui relate des agissements de la sorte dans la Florence de l'époque ${ }^{2}$. Un autre dispositif actanciel prévaut cependant dans les récits de Camus, qui mettent le plus souvent en scène un individu qui est corrompu ou se corrompt. On y reconnaît bien sûr un lieu de la topique morale que souligne Camus dans ses hommages à François de Sales: «L'esprit humain par la corruption de la nature a tant de propension vers le mal» ${ }^{3}$.

Dans le cadre particulier du récit, le personnage corrompu advient à travers la désignation nominale qui sert à le constituer dans son individualité, mais aussi à le décrire dans certains de ses aspects. En comparaison à la qualification opérée par attribution, les caractéristiques lexicalement impliquées dans le terme désignatif apparaissent inhérentes au personnage qui, fût-ce de manière accidentelle et transitoire, existe à ce titre. On peut alors se demander si les agents, ainsi lestés de qualités, se prêtent d'emblée à une saisie moralisante ou si, à l'inverse, de leurs actes procède une nomination axiologique qui sanctionne leur état de manière a posteriori. Mais toujours, la désignation finit par fixer l'état moral de l'agent, composantes indispensables des nouvelles camusiennes dont la visée édifiante tient dans une mesure importante de la dénonciation d'actions immorales.

Prenons pour premier exemple, qui nous servira tout au long de l'étude, un cas extrême, La Fureur Brutale (IV, 15) ${ }^{4}$; la pièce relate

2. J.-Cl. Waquet, De la corruption.

3. J.-P. Camus, L'Esprit du bien-heureux François de Sales, t. III, p. 130.

4. Le recueil des Evenemens singuliers (1628) se divise en quatre parties; nous l'indiquons par le chiffre romain suivi du numéro de la nouvelle. 
l'histoire d'un paysan ivrogne qui en vient à décimer sa famille pour finalement se donner la mort. La première désignation du personnage central de l'histoire paraît, de prime abord, neutre sur le plan sémantique: "un Villageois qui portoit le nom d'Adam» (756). Mais une introduction au récit, qui en fournit la clé herméneutique, demande à charger la description lexicale d'une signification précise:

Les moyens de faire mal leur [aux Rustiques] tombans entre les mains, on les voit aussi tost plongez dans les extremitez, du larcin, de l'yvrongnerie, de la cruauté, $\&$ de la fureur, avec tant de rage qu'ils semblent que sous un visage humain ils cachent un cœur plus sauvage que les plus farouches animaux (755).

Le personnage fait son entrée dans le récit en tant que villageois, habitué aux manières peu civilisées de la campagne. La désignation initiale pointe ainsi un être foncièrement délétère, inférieur à certains égards à l'animal. Et le nom propre qui lui est attribué cristallise, par écho biblique, le trait spécifique de la classe des «rustiques» en caractéristique individuelle et identitaire: il incarne la faute. L'assignation des qualités du paysan type à l'individu est confirmée dès la prédication qui suit l'introduction du personnage: "Cettui-ci estoit un homme dissolu \& de fort mauvaises mours" $(756)^{5}$. Tout est dit, le personnage n'a plus qu’à accomplir son rôle.

Tout au long du récit, et cela jusqu'aux premiers meurtres, l'identité est relayée très régulièrement par des pronoms anaphoriques ( $\mathrm{il}$ », «luy»). Ces formes pronominales instaurent un fonctionnement référentiel particulier, qui ne se résume pas au renvoi à un antécédent dont elles seraient les substituts transparents:

$I L$ désigne un référent en continuité avec une situation manifeste dans laquelle le référent se trouve impliqué comme actant principal. En termes instructionnels, il invite à chercher un référent, non pas simplement manifeste ou déjà connu [...] mais un référent qui est saisi dans un état de choses manifeste dont l'énoncé-hôte du pronom constitue un prolongement ${ }^{6}$.

5. L'incarnation de la classe sera encore rappelée par le moyen d'une expression générique: «ceste somme assez bonne pour un homme de sa sorte» (757). L'individu s'avère bien le représentant exemplaire d'un groupe social.

6. G. Kleiber, Anaphores et pronoms, p. 83. 
Le pronom ne reprend donc pas simplement un nom, mais recouvre l'image du personnage que l'ensemble du discours a construite. La désignation initiale, premier maillon de la chaîne, participe logiquement à cette élaboration; mais elle n'est pas seule et s'accompagne au moins d'une prédication, ainsi que, dans notre exemple, des désignations et prédications ultérieures. Le pronom opère ainsi la synthèse de tous ces éléments d'information. Malgré sa neutralité conceptuelle, le pronom se charge donc, tout au long de la nouvelle, d'un héritage sémantique croissant, que chaque emploi pronominal vient actualiser et développer prédicativement: "Avec ces vices il estoit malaisé qu'il fust bon mesnager» (756), «il n'estoit que mestayer» (ibid.), «il se va enfoncer dans une taverne» (757), «il trouva des frippons» (ibid.), etc. Procédant selon une logique du cumul, la désignation du paysan l'enferme dans sa destinée tragique. L'acte meurtrier apparaît finalement comme la conséquence inéluctable de la nature altérée du villageois:

Saisi d'une rage diabolique $\&$ desnaturee, il l'esgorgea [sa fillette] sur le champ, comme aussi celui qui [son fils] lui avoit demandé du pain. Et non content de ce massacre, il en fit autant à un innocent qui dormoit paisiblement dans le berceau (757-8).

Le système désignatif, très régulier jusqu'au moment crucial du crime, enregistre ensuite, dans les dernières lignes de la relation, trois variations lexicales:

Ce tygre se ruant sur elle luy coupa en mesme temps la gorge \& la parole (758).

Ce brutal l'alloit encore sacrifier à sa fureur (ibid.).

On trouva ce miserable veautré dans son sang (ibid.).

Uniformément, un syntagme nominal démonstratif actualise le référent. Ce processus, déclenché par le déterminant («ce»...), consiste à pointer directement, sans tabler sur un calcul sémantique, un objet particulièrement saillant dans le discours. Autrement dit, le démonstratif assure, sans impliquer un discernement de catégories conceptuelles, l'identification de l'objet pertinent. Ainsi, le contenu nominal, dès lors qu'il n'intervient pas dans le mécanisme de repérage, constitue une donnée de sens supplémentaire qui peut, tout au plus, valider la saisie du référent approprié. Par conséquent, si la composante nominale du syntagme 
démonstratif ne participe pas directement à l'identification du bon référent, le choix lexical s'avère, pour l'instance auctoriale, faiblement contraint:

[...] cet élément lexical, ou descripteur, peut être exploité de manière relativement libre pour (re)catégoriser le référent, à des fins planificatoires, interactives et/ou argumentatives très diverses 7 .

Et il en va bien ainsi des trois expressions utilisées par Camus. La première ("ce tygre») suppose, vu sa dimension métaphorique, que la cible référentielle soit préalablement fixée, afin que la catégorie conceptuelle, inédite et illogique dans sa signification littérale, puisse être adéquatement attribuée. De fait, on se souvient que le lien de similitude entre le paysan et l'animal avait déjà été signalé: «ils [les rustiques] cachent un cœur plus sauvage que les plus farouches animaux" (755). La plus-value de sens, inhérente à la métaphore, s'impose et donne à comprendre que le paysan intègre, dans son être même, une part de nature animale et sauvage.

Dans les deux autres désignations démonstratives, les éléments nominaux manifestent également, compte tenu de leur visée évaluative, la liberté qui a présidé à leur choix. En outre, les deux noms résultent d'une substantivation d'adjectifs («brutal», «misérable»). Pour que soit justifiée leur nominalisation, il convient que les adjectifs dénotent des qualités permanentes dans le comportement décrit. La forme nominalisée ne sert alors plus à caractériser l'individu sur le mode attributif, en lui assignant une propriété, mais à le constituer intrinsèquement dans sa réalité qualitative la plus essentielle. Un trait moral du personnage, en raison de sa constance remarquable, est ainsi érigé en détermination substantielle. Mais voyons de plus près en quoi consistent ces caractéristiques que Camus fixe par l'acte de nomination. Dans la terminologie de Jean-Claude Milner, les adjectifs «brutal» et «misérable" sont dits "non-classifiants»: cela signifie qu'ils se trouvent, étant donné leur charge affective, aptes à intégrer une tournure exclamative ("Quel brutal/misérable!») et qu'ils ne délimitent pas, hors de leur énonciation particulière, un ensemble d'individus susceptibles de former une catégorie à part entière (les brutaux et les misérables ne sont pas

7. M.-J. Béguelin, «L'usage des SN démonstratifs dans les Fables de La Fontaine», p. 96. 
répertoriés). Ainsi précise Milner, «lorsqu'on les emploie [les adjectifs non-classifiants] comme attributs dans une phrase du type "X est...», il est difficile de l'interpréter comme un jugement d'appartenance» ${ }^{8}$. Par conséquent, Camus substantialise des propriétés par la désignation qualitative, mais il soustrait également le personnage à la typologie usuelle des caractères. Le paysan qui se trouve inscrit dans une catégorie morale inédite, faite sur mesure, se voit finalement consacré dans sa nature exceptionnelle, en l'occurrence infâme et corrompue. Ce sort, Camus le confère en partage à tous les représentants de la classe paysanne. Dans une autre nouvelle, il en décrit un ressortissant dans ces termes:

Un paysan, qui n'ayant rien de l'homme que le visage, avoit l'humeur aussi farouche $\&$ cruelle que les bestes sauvages, parmi lesquelles il demeuroit (IV, 6: 687).

Hors du commun, mais aussi rejeté à l'extrême négatif sur l'échelle de l'évaluation morale, le campagnard apparaît, dans l'anthropologie camusienne, comme un être a priori corrompu. C'est, outre dans les touches descriptives, par la logique désignative que nous est donné à voir un être naturellement avili: le cadrage évaluatif qui accompagne la première saisie référentielle et qui se trouve maintenu tout au long du récit par la récurrence pronominale confine le rustique, avant même qu'il n'en convienne par l'action, dans un destin néfaste.

D'autres personnages, diversement inscrits dans l'organisation sociale, témoignent d'un processus de corruption bien différent de celui incarné par le paysan. Ainsi en va-t-il, autre exemple qui nous servira pour la suite de l'analyse, de Démétrie, femme de mauvais conseil (Le Conseil Chastié II, 13). Par sa conduite, le personnage de Démétrie engage la nature féminine dans son entier, fonds atavique sur lequel sont prises les décisions de l'individu. Un avertissement en préambule au récit définit, bien au-delà du cas individuel, l'étendue des enjeux que l'anecdote engage:

Ce n'est pas d'aujourd'hui que les conseils des femmes, principalement des mauvaises, sont dangereux. A la naissance du monde, le premier de tous les hommes faillit par le conseil de sa femme, \& nous payons tous

8. J.-Cl. Milner, De la syntaxe à l'interprétation, p. 299. 
les jours les interest de ce mauvais conseil, par lequel nous pouvons dire que le peché s'est introduit au monde (385).

On appréciera la modalisation du jugement («principalement des mauvaises»), qui ne tient pas ici de la simple prudence rhétorique. Au moment de l'entrée en scène, la jeune fille est nommée sans implication de considération morale: "Une Damoiselle que nous appellerons Demetrie». Elle ne se trouve donc pas d'emblée affectée par une tare, et cela malgré les maux endurés par elle en raison d'un mariage forcé avec un vieillard «jaloux» et «imbécile». Mais une transformation s'opère lorsque, veuve, elle obtient sa délivrance:

S'enyvrant de son propre tonneau, elle se prit si avidement à la liberté, qu'elle en changea l'usage en abus, $\&$ de prisonnière honorable elle devint une infame libertine (385).

Deux prédicats transformateurs sont exprimés successivement. Un verbe d'action ("changer l'usage de la liberté en abus») signale une mutation dans l'ordre pratique. Au changement d'habitude fait suite l'expression d'une altération qui signale l'inscription de l'individu dans une catégorie dépréciative ("elle devint une infame libertine»), en contraste avec le mérite initial ("prisonnière honorable»). Il a donc fallu que le personnage en premier lieu agisse pour que le récit formule une sanction morale et l'enregistre dans un nouvel état.

Et l'évolution de Démétrie se développe et se radicalise, en conformité à un principe qui vaut comme explication métanarrative: "personne ne devenant meschant tout à coup" (386). Outre les termes anaphoriques ("elle», «lui», «la») qui maintiennent l'identité de l'individu tout en l'associant à diverses considérations prédicatives, plusieurs noms désignent en faisant sens par eux-mêmes. En effet, une fois accompli le premier forfait, tous les désignateurs nominaux qui se rapportent à la protagoniste concourent, dans la suite du récit, à une évaluation morale. La plupart sont des descripteurs nominaux auxquels tendent à s'associer des qualifications: "ceste specieuse, mais devorante Panthere» (387), "la rusee femelle (388), «ces mal-heureuses femmes; ceste perduë; ceste infidelle; ceste pipeuse beauté» (390), "ceste femme dont il estoit affollé» (391); "cette mauditte femme; la mauvaise conseillere» (392). On remarquera que, lorsqu'un adjectif s'insère dans le syntagme nominal, il se place dans tous les cas en antéposition au nom. Ainsi amenée, la propriété ne 
constitue pas un ajout, sur le mode de l'annexe, à la désignation, mais participe intégralement à la constitution du référent. En première place dans le segment, l'adjectif signale l'aspect sous lequel doit être envisagé l'objet non encore livré et fonde ainsi, en pleine solidarité avec le nom qui

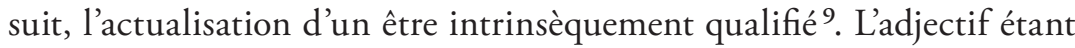
alors systématiquement porteur d'axiologie, il se rattache à un substantif qui peut rester neutre sur le plan de l'évaluation morale ("femelle», «femme», «beauté », "conseillère»). Mais dès que l'épithète disparaît, la signification morale investit le nom par le moyen (commenté plus haut) d'adjectifs substantivés: "ceste perduë»; "ceste infidelle»; la personne, disions-nous, incarne alors la qualité qui est présumée foncière et stable en elle. Des noms propres, autres que celui introduit en début de récit, servent également à identifier l'agent: «ceste Circé» (386 et 390), «ceste impudique Phriné» (387); "ceste Laïs» (388); "ceste Thays» (390). Les noms propres font ici l'objet d'un emploi métaphorique, dans la mesure où ils désignent à neuf un personnage qui ne portait pas, auparavant, le nom servant en l'occurrence à sa désignation. La reconnaissance de l'individu visé ne peut donc pas être assurée par le nom, qui ne respecte pas les conventions baptismales; Marie-Noëlle Gary-Prieur le confirme dans un commentaire consacré au syntagme "ce» NP: «l'effet essentiel du démonstratif sur le nom propre est en somme de décharger ce dernier de sa fonction identifiante " ${ }^{10}$. Il en va bien ainsi, car le déterminant démonstratif est apte, avons-nous souligné, à résoudre par lui-même le repérage référentiel dans le contexte discursif. Camus a donc tout loisir de recourir, dans le dispositif référentiel, à des noms propres qui ne servent pas à confirmer l'identification présupposée par le déterminant, mais à signifier à propos de l'individu concerné. Il sélectionne par conséquent, dans l'encyclopédie, des noms propres chargés de connotations et les applique à son personnage. Il en résulte une qualification qui ne procède pas sur le mode de l'attribution d'une propriété, mais par un déplacement identitaire: le personnage de Démétrie est métaphoriquement identifié à un autre qui vaut comme symbole et dont les valeurs associées se trouvent nouvellement endossées. La protagoniste de l'histoire se confond donc avec des figures quasi allégoriques, qu'elle réactualise et

9. Pour M. Cressot (Le Style et ses techniques), l'épithète antéposée faisant corps avec le substantif marque une qualité constitutive, fondamentale, inhérente à l'objet.

Io. M.-N. Gary-Prieur, Grammaire du nom propre, p. 214. 
fait revivre à sa façon: une magicienne païenne, une hétaïre, une amante et une pécheresse célèbres recomposent ainsi l'identité de Démétrie. L'intérêt du nom propre, par rapport au descripteur lexical, réside en ce qu'il ne donne pas à voir l'individu de manière partielle, sous un angle choisi, mais le saisit globalement dans son unité qui intègre la somme ouverte de ses qualités. En recourant à des "noms propres sémantisés", Camus exprime la radicalité de la transformation morale du personnage qui n'est pas affecté superficiellement, mais intégralement, dans son unité personnelle: Démétrie n'est, littéralement, plus la même.

Au regard des deux cas retenus, nous constatons que l'état de corruption ne s'exprime pas à l'identique pour toutes les figures malfaisantes que répertorie Camus dans ses nouvelles. Le paysan souffre d'une nature délétère qui lui vaut, avant même d'apparaître dans des circonstances, d'être fixé par une désignation qui entérine son immoralité de principe. En comparaison, la femme ne subit pas, de manière aussi univoque, une sanction morale a priori ${ }^{11}$. Elle traîne certes des antécédents bibliques peu favorables (Eve), mais ces déterminations restent latentes aussi longtemps qu'un acte délictueux ne les a pas ravivées; alors seulement tombe un jugement irrévocable, qui se cristallise, comme nous l'avons vu, dans le nom. Ces considérations nous incitent à distinguer, dans les représentations camusiennes du sujet humain, une corruption par nature ou essentielle, qui se manifeste d'office, et une corruption par accident, plus aléatoire, qui est avérée uniquement dans la mesure où les circonstances le veulent.

A ce stade, deux paramètres nous ont servi à distinguer des modalités du comportement, l'un socio-professionnel (paysan), l'autre sexuel (féminin). Cependant ces traits ne s'excluent pas et peuvent, en d'autres situations, cohabiter en un même individu; dans un contexte donné, la paysanne n'agira donc pas tout à fait comme le paysan. Et Camus de développer, de nouvelle en nouvelle, une caractériologie en diversifiant les déterminations qu'il signale pour ses personnages: l'origine nationale, la naissance (noblesse, haute ou petite bourgeoisie, peuple), l'âge, la

II. Des considérations plus radicalement critiques apparaissent dans une autre pièce du recueil, La Chasteté Courageuse (III, 12): «Il n'y a rien de plus infirme que ce sexe [féminin], à qui la debilité est escheuë en partage» (547). Ces propos visent cependant surtout à mettre en valeur l'attitude morale de la protagoniste. 
confession, etc. ${ }^{12}$ On découvre alors que le débordement colérique s'accomplit différemment chez les paysans qui sont des "personnes rudes $\&$ qui n'ont rien d'humain que le visage» ${ }^{13}$; chez Ménalque qui nous apprend par son comportement que "c'est le propre des Soldats de prendre feu comme une poudre seche à la premiere estincelle, \& de se mettre en colere lors qu'on les fasche ${ }^{14}$; chez le Sicilien Florian dont l'humeur jalouse allume "dans le cour un furieux appetit de vengeance» ${ }^{15}$; ou encore chez Peregrin et les Allemands qui, "quoy qu'habitans d'un climat froid, sont neantmoins prompts $\&$ boüillans» ${ }^{16}$. Il s'agit là d'une collection d'individus potentiellement furieux dont les débordements passionnels sont mis en perspective à travers un ensemble variable de traits caractérisants. C'est donc bien, comme nous avons pu le montrer ailleurs, sur la base d'un système de facteurs que se constitue l'anthropologie fondatrice de la poétique camusienne:

Le personnage camusien accède à l'individualité par la composition plus ou moins originale et spécifiante de principes généraux. Produit d'un système combinatoire, il est donc saisi [...] à la fois sous l'angle de la singularité que requiert l'actualisation diégétique et sous celui de la généralité fondatrice de lois morales ${ }^{17}$.

Une combinatoire plus ou moins fine des qualités génère en définitive des variations individuelles qui font dire à Camus que "tous ne sont pas jettez en mesme moule» (1630a: 445). Nous voyons ainsi le processus de corruption se réaliser de multiples façons et à différents degrés selon les composantes du caractère qui définissent les personnages mis en scène.

I2. De manière analogue, La Mesnardière fera se croiser, dans sa Poétique (1640), un catalogue des passions avec les cinq paramètres suivants: l'âge, la fortune présente, la condition de vie, la nation et le sexe.

13. J.-P. Camus, Les Spectacles d'horreur, p. 453.

I4. J.-P. Camus, Les Rencontres funestes, p. 8.

I5. J.-P. Camus, L'Amphitheatre sanglant, p. 354.

16. Ibid., p. 452.

I7. J. Zufferey, Le Discours fictionnel, p. 151. 


\section{L'intention et la responsabilité}

Nous avons vu que la narration, par les formes désignatives, saisit l'agent, que ce soit dans une nature invariante pour le paysan ou dans des états évolutifs mais congruents avec ses actions pour le personnage féminin. Outre la dénotation des personnages, le récit règle également les conditions dans lesquelles les procès se déclenchent, voire s'enchaînent les uns aux autres. Sur ce point, Françoise Revaz nous invite à distinguer entre les événements qui adviennent par le truchement d'une cause externe («la neige fond sous l'effet calorifère du soleil») et les actions intentionnelles que commet un sujet anthropomorphe qui poursuit un but ("mentir pour éviter une punition»). Revaz précise que, entre ces deux extrêmes, des modalités du faire impliquant des degrés de conscience variés (avec préméditation, volontaire, involontaire) se déploient et définissent l'attribution des responsabilités:

L'expression de l'intention est le signe le plus manifeste de la prise en responsabilité de l'action par un agent. Des notions comme celles d'«homicide involontaire» ou d'«imprudence» montrent que le degré de responsabilité est évalué proportionnellement au degré d'intentionnalité ${ }^{18}$.

En maintenant notre attention sur les formes de l'expression narrative, nous proposons d'examiner la force d'implication des sujets dans leurs actions néfastes. Il s'agit, plus exactement, de dégager le niveau de responsabilité que le narrateur camusien met à la charge des instances malfaisantes qu'il dépeint. En prenant appui sur les deux cas exemplaires du paysan ivrogne et de la femme de mauvais conseil, nous allons mettre en évidence la dimension plus ou moins volontaire de l'action menée par chacun des agents.

Dans les deux anecdotes funestes, un contexte particulier accueille l'action et, dans une certaine mesure, l'oriente vers sa fin. Ainsi les circonstances géographiques contribuent d'emblée à limiter les possibles narratifs:

L'Alsace est une des meilleures \& plus fertiles provinces de l'Allemagne, \& si voisine des Suisses, qu'elle contient un de leurs plus

I8. F. Revaz, Introduction à la narratologie, p. 30. 
celebres cantons, qui est celui de Basle. Là croist cet excellent vin de Rhin. (756)

En une ville de Sicile, qui n'est point nommee dans la Relation Italienne, une Damoiselle que nous appellerons Demetrie, fut donnee pour femme à un vieillard en un âge fort tendre, la ialousie \& l'imbecillité de cet homme caduc luy osterent toute sorte de plaisir en ce mariage (385).

Les conditions climatiques, qui régissent les activités locales, les conditions de vie, mais aussi les coutumes et les humeurs, sélectionnent in abstracto des sénarios narratifs privilégiés: l'ivrognerie et ses conséquences conviennent aux contrées ensoleillées d'un pays viticole; alors que l'accoutumance - dont Camus soutient qu'elle est « une autre nature ${ }^{19}$ - favorise dans la péninsule italienne l'émergence d'un tempérament jaloux et vengeur ${ }^{20}$. Ces déterminations du caractère, plutôt a priori et dogmatiques, sont accompagnées dans le récit camusien de considérations plus conjoncturelles. Aussi nous intéresse-t-il de cerner et analyser le processus par lequel le déclencheur du récit, vecteur initial vers la faute, se trouve introduit dans l'histoire. Dans la relation de la femme libertine, un élément décisif, outre le mariage et la mort du vieux jaloux, intervient de façon inopinée dans le cours des événements:

Parmi ceux qui abbordoyent ceste specieuse, mais devorante Panthere, il y eut un Seigneur de marque, dont la qualité \& les moyens autant que la gentillesse lui donnerent dans les yeux (387).

C'est par un simple constat de fait objectif qu'est introduit dans l'histoire le personnage par lequel le dénouement funeste va se produire. La formule impersonnelle ( il y eut») instaure, en rupture avec l'amont du récit, une situation nouvelle qui n'apparaît dépendre d'aucun calcul ou menée individuelle. Sans lien de reprise anaphorique avec les composantes narratives déjà installées, et donc sans raison manifeste, le personnage fait une entrée imprévue. Ce déficit d'explication soustrait

19. J.-P. Camus, Les Evenemens singuliers, p. 261.

20. Notons que deux accès de jalousie sont mentionnés en titre de nouvelles dans Les Spectacles d'horreur: «La jalousie precipitée» et "La jalouse Fureur»; les deux histoires se déroulent dans la péninsule italienne. Camus relève également, dans une nouvelle des Evenemens singuliers: "L'Italie est assez descriee parmi les autres nations pour l'incontinence» (p. 127). 
la nouvelle donnée narrative du domaine d'influence et de maîtrise des protagonistes en place. Démétrie, la figure centrale de l'histoire, est donc prise dans un contexte qui va orienter son action, mais sur lequel elle n'exerce, malgré ses efforts, que peu d'influence.

On pourrait croire qu'un dispositif analogue lance l'aventure malheureuse du paysan:

Un iour il avint qu'Adam estant allé à la ville, receut de son Maistre, dont il n'estoit que mestayer, un quartier de son salaire (756).

La construction impersonnelle ( il avint») semble signaler un aléa de la vie ou l'intervention d'une force supérieure. Mais à la différence de la libertine, la paysan a, dès le début, œuvré de manière à faire évoluer les événements conformément à ses visées: "Il representa si bien ses necessitez \& les besoins de sa pauvre famille...» (756-7). Le verbe d'action («représenter») est de nature intentionnelle, dans la mesure où il présuppose un vouloir de la part du sujet. Le paysan se trouve par conséquent à l'origine de la situation dans laquelle il va commettre ses crimes. En définitive, les deux protagonistes ne participent pas à part égale à la mise en place de leur forfait, la femme subit («lui donnerent dans les yeux») alors que le rural agit.

Ces attitudes différenciées ne correspondent cependant pas à une répartition définitive des responsabilités. Une fois l'action engagée, le comportement de chacun évolue en quelque sorte à l'inverse de celui de l'autre. Plongée dans une situation inattendue, Démétrie manigance pour en tirer tous les profits possibles. Elle s'adonne ainsi à des stratégies auxquelles président des intentions malveillantes que le récit explicite en plusieurs occasions; en voici quelques exemples: «son dessein estoit de donner de l'amour à plusieurs" (386); «la resolution qu'elle avoit prise de se donner du bon temps» (ibid.); "desireuse au contraire de faire servir ce don precieux de la liberté au desreglement de ses appetits" (ibid.); "elle mit en œuvre tous ses artifices pour faire en sorte que...» (388); "apres avoir donc employé inutilement tous ses artifices pour reconquerir son esprit» (391) ; "venir mieux à bout de son sanglant dessein" (ibid.). Les choix volontaires qu'effectue la protagoniste servent de vecteur principal à la progression du récit. Ainsi les actions qui s'enchaînent dans l'histoire manifestent une causalité narrative que dirigent les intentions avérées de la protagoniste. Jusqu'au dénouement final, qui lui échappe, les faits se 
déroulent conformément aux vues de Démétrie dont la conscience d'agir mal, et partant la responsabilité face à sa propre corruption, apparaît entière.

Soulignons comparativement que le campagnard alsacien, une fois le cours des événements lancé, assume peu d'actions à titre volontaire. Même la prise de décision, présentée comme telle, échappe au contrôle de sa raison et par conséquent à sa maîtrise personnelle:

Le diable lui ietta un tel desespoïr dans le cœur, qu'il resolut de finir par un licol sa miserable vie. Sur ceste mortelle determination il entre chez lui, avec des yeux hagards \& un visage affreux (757).

Le choix opéré par l'individu («il resolut»), exprimé en place de subordination syntaxique, dépend d'une donnée principale ( «le diable lui ietta un tel desespoïr dans le cœur»). Le lien de dépendance entre les deux termes relève de la conséquence forcée, ce que déclenche le marqueur d'intensité "tel». Jean-Michel Adam a relevé la fréquence de ces structures corrélatives dans certains récits brefs du XVII ${ }^{\mathrm{e}}$ siècle et les commente ainsi: "L'adverbe [ou adjectif] intensif de la protase exprime un degré d'intensité à partir duquel la conséquence ne peut que se produire ${ }^{21}$. En l'occurrence la décision, contrainte par un paramètre externe à la sphère subjective du personnage (le diable), lui est littéralement imposée et apparaît donc inéluctable. C'est bien, finalement, la composante volontaire de la décision qui se trouve réduite, de sorte que le sujet perd, dans un acte pourtant éminemment intentionnel, la mầtrise de ses choix ${ }^{22}$. D'autres formulations, comme les participes passés passifs, signalent que le personnage ne commande plus la direction des événements, ni d'ailleurs ses états intérieurs les plus intimes:

Abandonné de Dieu \& tombé en sens reprouvé, il fut saisi de la brutale fureur que vous allez lire (757).

2I. J.-M. Adam, U. Heidmann, Textualité et intertextualité des contes, p. 250.

22. J.-M. Adam relève, à propos des Contes de Perrault, que les «formes intensives confèrent aux personnages des propriétés hors du commun" (ibid.). Relativement aux histoires tragiques de Camus, nous pouvons émettre un constat exactement inverse: les corrélatives intensives retirent au personnage ses compétences les plus élémentaires à agir par lui-même et l'enferment dans des schémas mécanistes. 
Saisi d'une rage diabolique $\&$ desnaturée, il l'esgorgea sur le champ (757-8).

Investi, par la tournure passive, du rôle actantiel de patient, le personnage abandonne les prérogatives de l'agir à cela même que sa raison chrétienne devrait soumettre (le complément d'agent introduit par la préposition "de»). Cédant à ses émotions, le sujet développe une relation altérée à lui-même:

Adam s'en retournant s'alloit esgarant en ses pensées, dans lesquelles il se perdoit comme dans un labyrinthe (757).

La réflexion sur soi, qui n'est certes pas abolie (voir la tournure pronominale réfléchie), opère désormais sur le mode de la confusion, de l'égarement et, à terme, de la folie. Le sujet a donc perpétré une série de crimes qu'il n'a pas choisi, volontairement, de commettre mais dont il a été, en raison de sa nature essentiellement corrompue, l'instrument adapté. Que la volonté du paysan ne soit que partiellement engagée ne constitue nullement, aux yeux de Camus, une circonstance atténuante: dans sa perspective, qui relève de la sotériologie plutôt que du droit, les forfaits du paysan alsacien, tout comme celui de son homonyme originel, affectent le genre humain dans son ensemble, jusqu'à rendre "l'homme tout à fait indigne des voyes \& misericordes eternelles» (758). Dans les deux anecdotes retenues, la mort finalement sanctionne des destins qui, chacun de manière spécifique, ont cédé à la corruption.

$\mathrm{Au}$ terme de ces quelques observations, très ponctuelles dans une œuvre pourtant immense, nous voulons surtout retenir que la corruption et la faute constituent les concepts centraux de la poétique camusienne. Cela apparaît explicitement sur le plan thématique, où les figures tragiques que met en scène Camus partagent, du fait même de leur déchéance morale, un destin malheureux. Pour reconnaître à ces déterminations éthiques des personnages une fonction véritablement poétique, il resterait à montrer qu'elles conditionnent aussi la mise en intrigue du récit. Nous n'avons guère insisté ici sur le fait que le caractère de l'agent représente le principe régulateur de la configuration narrative, que le muthos est fonction de l'ethos. D'autres l'ont relevé avant nous: Anne de Vaucher Gravili, par exemple, insiste sur la structure des histoires tragiques qui s'articulent invariablement autour d'une 
transgression et d'une punition ${ }^{23}$. Plutôt que de chercher à dégager le schéma narratif propre au genre, ce qui ne servirait guère à caractériser une écriture singulière, il nous a semblé préférable de faire porter l'observation sur d'autres aspects discursifs révélateurs de la manière camusienne de raconter. Les formes linguistiques de la désignation et leur évolution au cours du récit, ainsi que le traitement discursif de l'intentionnalité participent, selon nous, à la construction éthique des personnages et permettent à Camus de cerner, par la collection des nouvelles, les variables du comportement humain: le profil de chaque intervenant, composé d'un ensemble de traits caractéristiques, induit des écarts de comportement systématiques ${ }^{24}$. A l'échelle du recueil ou de l'œuvre, prend ainsi forme une vaste enquête sur les ressorts de l'agir humain qui, par sa portée, confine à l'investigation anthropologique. En effet l'auteur invite, en racontant, à saisir les lois générales du disfonctionnement pathique dont résultent, comme énoncé en notre exergue, les formes de la corruption humaine.

Joël ZuFFEREY

Université de Lausanne

23. A. de Vaucher Gravili, Loi et transgression.

24. Nous avons exposé ailleurs la logique sous-jacente aux dérèglements des actions humaines (J. Zufferey, "De l'occasionnel au sériel» et Le Discours fictionnel, p. 118126). 


\section{BIBLIOGRAPHIE}

\section{Textes}

Camus, Jean-Pierre, Les Diversitez de Messire Jean Pierre Camus Evesque \& Seigneur de Belley, t. VI, Lyon, Jean Pillehotte, 1610.

—, L'Amphitheatre sanglant, Paris, Joseph Cottereau, 1630.

-, L'Esprit du bien-heureux François de Sales, Paris, Gaumes Frères, 1840 (1640).

—, Les Evenemens singuliers, éd. par Max Vernet, Paris, Classiques Garnier, 2010 (1628).

—, Les Rencontres funestes, Paris, J. Villery, 1644.

—, Les Spectacles d'horreur, Paris, Andre Soubron, 1630.

\section{Travaux}

Adam, Jean-Michel, Heidmann, Ute, Textualité et intertextualité des contes, Paris, Garnier, 2010.

BÉGuelin, Marie-José, "L'usage des SN démonstratifs dans les Fables de La Fontaine», Langue française, 120 (1998), p. 95-109.

Cressot, Michel, Le style et ses techniques, Paris, PUF, 1983.

Gary-Prieur, Marie-Noëlle, Grammaire du nom propre, Paris, PUF, 1994.

Kleiber, Georges, Anaphores et pronoms, Louvain-la-Neuve, Duculot, 1994.

Milner, Jean-Claude, De la syntaxe à l'interprétation, Paris, Seuil, 1978. Revaz, Françoise, Introduction à la narratologie: action et narration, Bruxelles, De Boeck \& Duculot, 2009.

Vaucher Gravili, Anne de, Loi et transgression: les Histoires tragiques au XVII siècle, Lecce, Milelle, 1982. 
WAQUET, Jean-Claude, De la corruption: morale et pouvoir à Florence aux XVII et eVIII e siècles, Paris, Fayard, 1984.

Zufferey, Joël, "De l'occasionnel au sériel: les histoires tragiques de Jean-Pierre Camus», Variations 7 (2001), p. 75-87.

-, Le discours fictionnel: autour des nouvelles de Jean-Pierre Camus, Louvain, Peeters, 2006. 
